\title{
Métodos de trabajo y resultados de refuerzos en madera de un forjado del siglo xviI de la Sacristía de San Miguel en Morón de la Frontera (Sevilla)
}

\author{
Working methods and results of timber reinforcements in a sub-floor \\ of the 17th-century sacristy of San Miguel in Morón de la Frontera (Seville)
}

\author{
I. Ariza López $^{(*)}$, J. M. Guerrero Vega ${ }^{(*)}$, F. Pinto Puerto ${ }^{(*)}$
}

\section{RESUMEN}

Durante el pasado siglo se pusieron en práctica numerosas soluciones de refuerzo de antiguos forjados de madera mediante losas que pretendían suplir las carencias de funcionamiento por causas reológicas o por deterioro de algunas de sus piezas. Algunas de estas soluciones, sin embargo, supusieron una sobrecarga innecesaria que agravó las patologías previas con el paso del tiempo, hasta el punto de requerir su apuntalamiento. Éste es el caso de uno de los forjados de la sacristía de la parroquia de San Miguel de Morón, un edificio levantado en el transcurso del siglo XVII anexo al templo. Los pisos de sus dos plantas estaban solucionados mediante forjados de vigas de madera y bovedillas de yeso. Como parte de los trabajos de restauración realizados en 2013, se desmontó un refuerzo previo realizado en 1970 mediante losa de hormigón, para ejecutar posteriormente un novedoso tipo de refuerzo con madera laminada, pretensando la unión entre las piezas nuevas y viejas, lo que ha permitido reducir de forma notable las flechas, mantener todos sus elementos principales y obtener un comportamiento más eficiente. La presente aportación expone las condiciones previas a la intervención, el proceso seguido y los resultados obtenidos durante esta restauración valorando los cálculos y previsiones realizadas.

Palabras clave: rehabilitación; patrimonio; refuerzo estructural; forjado de madera; encolado; pre-flexión.

\section{ABSTRACT}

During the 2oth century numerous solutions for reinforcing wood sub-floors with concrete slabs and frameworks were introduced with the aim of remedying malfunctions due to rheological reasons or the deterioration of some of the components. In certain cases, however, these solutions created an excessive load, and as time went by they aggravated the original pathologies, to the extent where props were required. This is precisely the case of one of the sub-floors in the sacristy that was abutted to the parish church of San Miguel in Morón during the 17th century. The sub-floors on both levels are made of timber beams and hollow plaster bricks. As part of the restoration works carried out in 2013, a concrete slab added for reinforcement in 1970 was removed and replaced with a new reinforcement made out of plywood, pre-tensioning the joints between the new and old pieces. This has significantly reduced the cambers, preserved all the principal elements and led to a more efficient performance. This paper describes the conditions prior to the intervention, the process followed and the results obtained during these restoration works. It also assesses the calculations and projections made.

Keywords: renovation; heritage; structural reinforcement; wood sub-floor; gluing; pre-flexion.

(*) Universidad de Sevilla (Sevilla, España).

Persona de contacto/Corresponding author: inigoariza@us.es (I. Ariza López)

ORCID: http://orcid.org/oooo-0oo2-3794-3550 (I. Ariza López); http://orcid.org/oooo-ooo3-3164-328X

(J. M. Guerrero Vega); http://orcid.org/oooo-0003-4608-6818 (F. Pinto Puerto)

Cómo citar este artículo/Citation: Ariza López, I., Guerrero Vega, J. M., Pinto Puerto, F. (2016). Métodos de trabajo y resultados de refuerzos en madera de un forjado del siglo xvi de la Sacristía de San Miguel en Morón de la Frontera (Sevilla). Informes de la Construcción, 69(545): e180, doi: http://dx.doi.org/10.3989/ic.16.041.

Copyright: (C) 2017 CSIC. Licencia / License: Salvo indicación contraria, todos los contenidos de la edición electrónica de Informes de la Construcción se distribuyen bajo una licencia de uso y distribución Creative Commons Attribution License (CC BY) Spain 3.o. 


\section{INTRODUCCIÓN}

Los forjados de madera realizados durante los siglos xvII y XVIII son probablemente uno de los elementos menos estudiados dentro del ámbito de la intervención en estructuras históricas (1). A esto contribuye que la madera es un material muy sensible a las condiciones de mantenimiento del edificio, siendo muy frecuente observar el deterioro en los extremos empotrados de las vigas, lo que ha llevado a adoptar soluciones de urgencia, a veces muy precarias. Además, las tensiones de trabajo de la madera están siempre mucho más cerca de su límite admisible, a diferencia de los materiales como la piedra o el ladrillo que trabajan a compresión, formando parte de muros. En consecuencia, el deterioro en la madera es más dramático que en otros materiales (2).

Estas circunstancias han llevado a la sustitución irracional de muchos de estos elementos constructivos por otros materiales, como el acero o el hormigón, que a la larga, además de adulterar el edificio, no han tenido un buen comportamiento (3). Por otro lado, las nuevas exigencias legales propician su obsolescencia, promoviendo su sustitución (4). Sin embargo, la madera ofrece amplias posibilidades de reparación y refuerzo con relativa facilidad.

Por ello es importante que en la intervención en estructuras históricas se utilicen técnicas adecuadas para el refuerzo o la reparación de los forjados de madera que permitan: conservar el funcionamiento estructural de la misma; mantener en la medida de lo posible el sistema constructivo-estructural original; reducir el impacto medioambiental que supone toda sustitución, y preservar su capacidad de reversibilidad y sostenibilidad (5).

Entre todos los materiales y técnicas existentes, la resinas epoxi han facilitado el desarrollo de métodos eficaces de reparación de estas estructuras. Podemos encontrar sistemas variados de reparación basados en ellas, ya sean de unión de madera con madera en los apoyos (6), a lo largo de la pieza aumentando la sección (7), o con refuerzos de metal o con varillas de fibra de vidrio (8). En los últimos años se han añadido nuevos productos, como la fibra de carbono (9), y nuevas técnicas de pretensado con los plásticos reforzados con fibras (10). Sin embargo, no existen referencias al uso de la pre-deformación para el refuerzo de madera en rehabilitación, salvo cuando las soluciones pertenecen al ámbito de la ingeniería, y combinan hormigón con acero (11).

En la intervención que exponemos aquí se ha combinado de manera novedosa el encolado y la pre-flexión con piezas de madera, consiguiendo más eficacia para el refuerzo de forjados, contribuyendo al cumplimiento de los requerimientos de reversibilidad, impacto reducido y sostenibilidad. Este tipo de refuerzo ha sustituido otro anterior realizado en los años setenta del siglo pasado y que consistió en el añadido de una losa de hormigón, cuyo peso generó un exceso de deformación que obligó al apuntalamiento del forjado en 1998.

\section{EL EDIFICIO. EVOLUCIÓN Y ESTADO DEL INMUEBLE PREVIO A LA INTERVENCIÓN}

El forjado intervenido forma parte de una edificación de tres plantas adosada a la cabecera de la iglesia de San Miguel Arcángel, en Morón de la Frontera (Sevilla, España) (Figura 1), que servía para alojar varias dependencias parroquiales.
Se construyó en diversas fases realizadas entre 1596 y 1625 que están bien documentadas (12), (13), (14), (15). En estas fechas la población había superado una dramática epidemia de peste, comenzando una recuperación económica que permitió iniciar la construcción de estas dependencias parroquiales. La traza de la cabecera correspondía a Vermondo Resta, entonces maestro mayor del arzobispado hispalense. Cuando restaba por concluir la capilla mayor y la sacristía, Lorenzo de Oviedo, maestro mayor de los alcázares sevillanos, proyecta hacer una nueva planta en 1601 respetando la traza de anterior. Apenas levantados los muros, murió Oviedo, por lo que la construcción del forjado que cubre la sacristía se demoró hasta 1605, a cargo del maestro albañil Francisco Quirós, quedando por hacer la segunda planta destinada a librería.

En 1611 visita el edificio el maestro mayor del arzobispado Miguel de Zumárraga, acompañado del autor de las trazas iniciales Vermondo Resta para supervisar las obras, que habían progresado esos años con renovado impulso, llegando a cubrirse en 1616 la segunda planta de la sacristía. El carpintero que trabajó sobre el forjado debió ser el mismo que realizó el mobiliario y las puertas de la sacristía en ese mismo año, Francisco Marín de la Torre. Quiere esto decir que entre 1605 y 1616 fue construido el forjado de bovedillas y vigas de madera sobre la sacristía.

Pocos años más tarde se elevó esta edificación en una nueva altura. Este proceso de reformas se prolongaría hasta 1726 , en el que será inaugurado definitivamente el templo. En 1755 y como consecuencia del terremoto de Lisboa, el edificio sufre numerosos desperfectos que serán subsanados en 1763 por el arquitecto Pedro Silva, quien actúa en los forjados de la sacristía sin que quede constancia del tipo de trabajo realizado. En la primera mitad del siglo xx una serie de reformas menores en la sacristía no afectaron al forjado. En la última etapa de reformas promovidas por la Dirección General de Bellas Artes, entre 1970 y 1983, se procedió al refuerzo del forjado, el cual tuvo que ser apuntalado en 1998 a consecuencia de la enorme flecha que presentaba y la rotura súbita de tres de sus vigas, manteniéndose en este estado hasta el año 2013, en el que se concluyeron los trabajos aquí descritos.

El forjado tiene planta rectangular de $14,5 \times 6,5 \mathrm{~m}^{2} \mathrm{y}$ una superficie construida de $94,25 \mathrm{~m}^{2}$. La sala a la que sirve de piso se encuentra sobre la sacristía del templo y cuenta con una altura libre de $4,5 \mathrm{~m}$.

\section{CARACTERÍSTICAS CONSTRUCTIVAS DEL FORJADO DE LA SACRISTÍA Y ESTADO DE CONSERVACIÓN}

El forjado está constituido por 18 viguetas de $17 \times 26,5 \mathrm{~cm}^{2}$ de escuadría (1 en Figura 2), separados sus ejes $75 \mathrm{~cm}$ aproximadamente, y que apoyan en el muro mediante canes moldurados. El entrevigado es de bovedilla de yeso y arena encajado sobre hendiduras en los laterales de las viguetas, con enlucido también de yeso ( 2 en Figura 2). El relleno (3 en Figura 2) cubre las viguetas y posee un espesor medio aproximado de $12 \mathrm{~cm}$, sin discontinuidad entre las bovedillas y el relleno, pues son básicamente el mismo material. Las viguetas cubren una luz de $654 \mathrm{~cm}$ y penetran en los muros entre 50 y $75 \mathrm{~cm}$.

Este tipo de forjado se corresponde con total exactitud con la descripción que realizó de Juan de Villanueva (16) (Figura 3): 


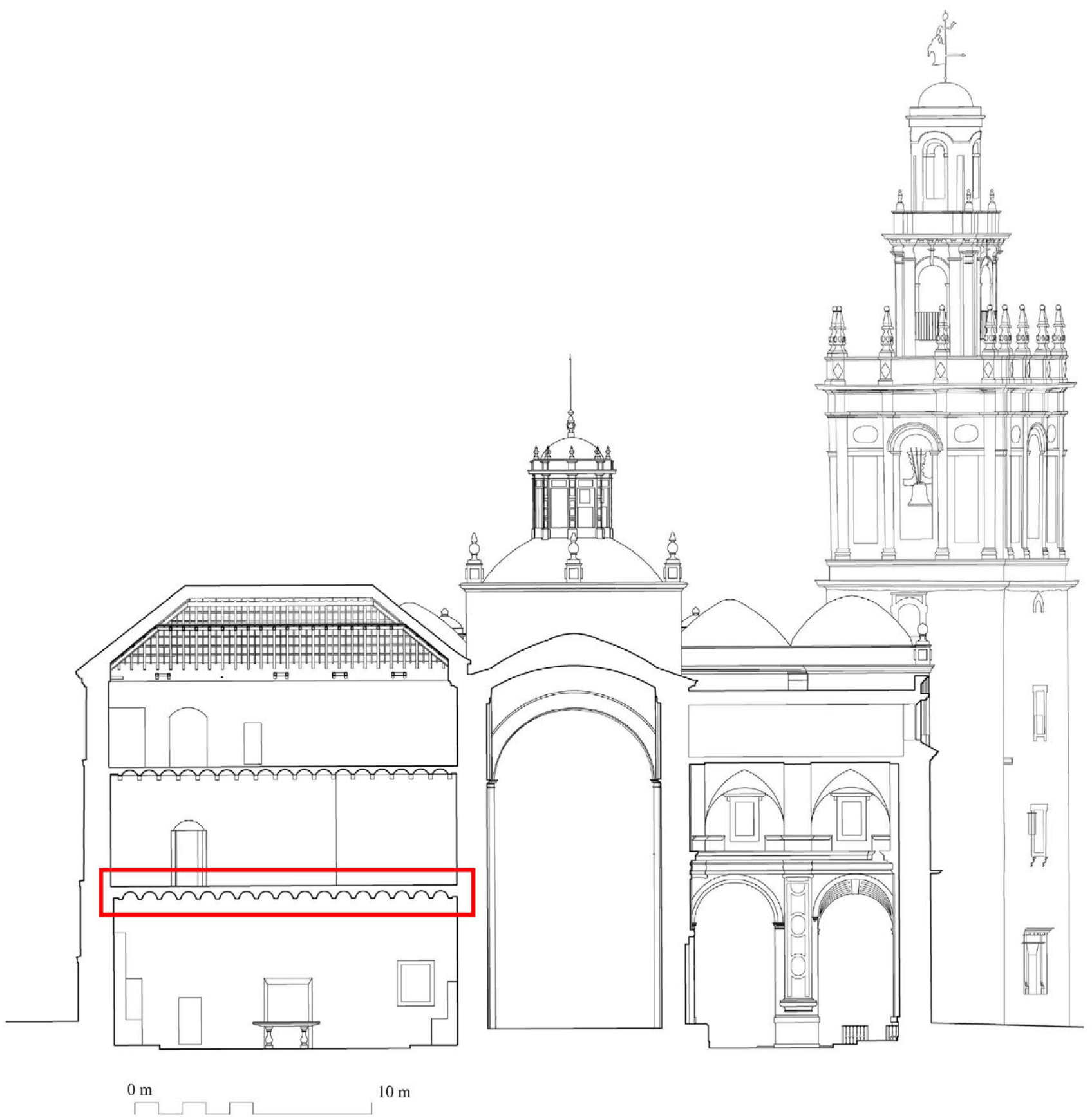

Figura 1. Sección de la sacristía y cabecera de la iglesia de San Miguel. Se ha marcado en rojo el forjado rehabilitado.

«Segundo, los que se forjan de bovedillas, que por arriba forman piso, y por abajo quedan descubiertas, así ellas como las maderas».

«Este (se refiere al forjado de bovedilla) se hace valiéndose de una formaleta llamada galápago (lám. IX, fig. 2), que se compone de dos maderillos unidos con goznes, que forman por arriba cuarto o círculo, poco más ó menos. Colócase ésta entre viga y viga á el alto que están hechas unas mochetillas en ellos, asegurándola de prestado con unos clavos, por encima se llena y forja el hueco que dejan hasta enrasar con las vigas con cascote menudo y yeso. Fraguado éste, se quita el galápago, y se pasa mas adelante, si de una vez no se forja toda la bovedilla. Lleno un vano, se pasa á hacer otro, y así sucesivamente se remata todo el suelo, y los galápagos dejan formado por abajo un cañon de bovedilla del ancho y vano que tienen las vigas entre sí. Este ancho suele ser variable, pues las vigas unas veces se colocan muy espesas, otras muy claras; pero la práctica del día en Madrid es de poco mas del grueso de la viga. En obras viejas se hallan de pie y medio, dos pies, y también de tres. Donde el yeso es de buena calidad y fortaleza, bien guarnecidas y blanqueadas las bovedillas, es obra que parece bien, y no carga ni acrecienta mucho el peso de los suelos».

En nuestro caso, sobre el relleno se disponía la solería original, según la estratigrafía que las catas ya mostraron durante los estudios previos a la intervención. Sobre este relleno se dispuso en los años setenta del pasado siglo una solera de hormigón armado de $8 \mathrm{~cm}$ de espesor medio y una armadura de mallazo de acero corrugado de $4 \mathrm{~mm}$ de diámetro cada 20 cm situada aproximadamente en su plano medio (4 en Figura 2) y sobre ella una solería de ladrillos cerámicos $14 \times 28 \mathrm{~cm}$ 


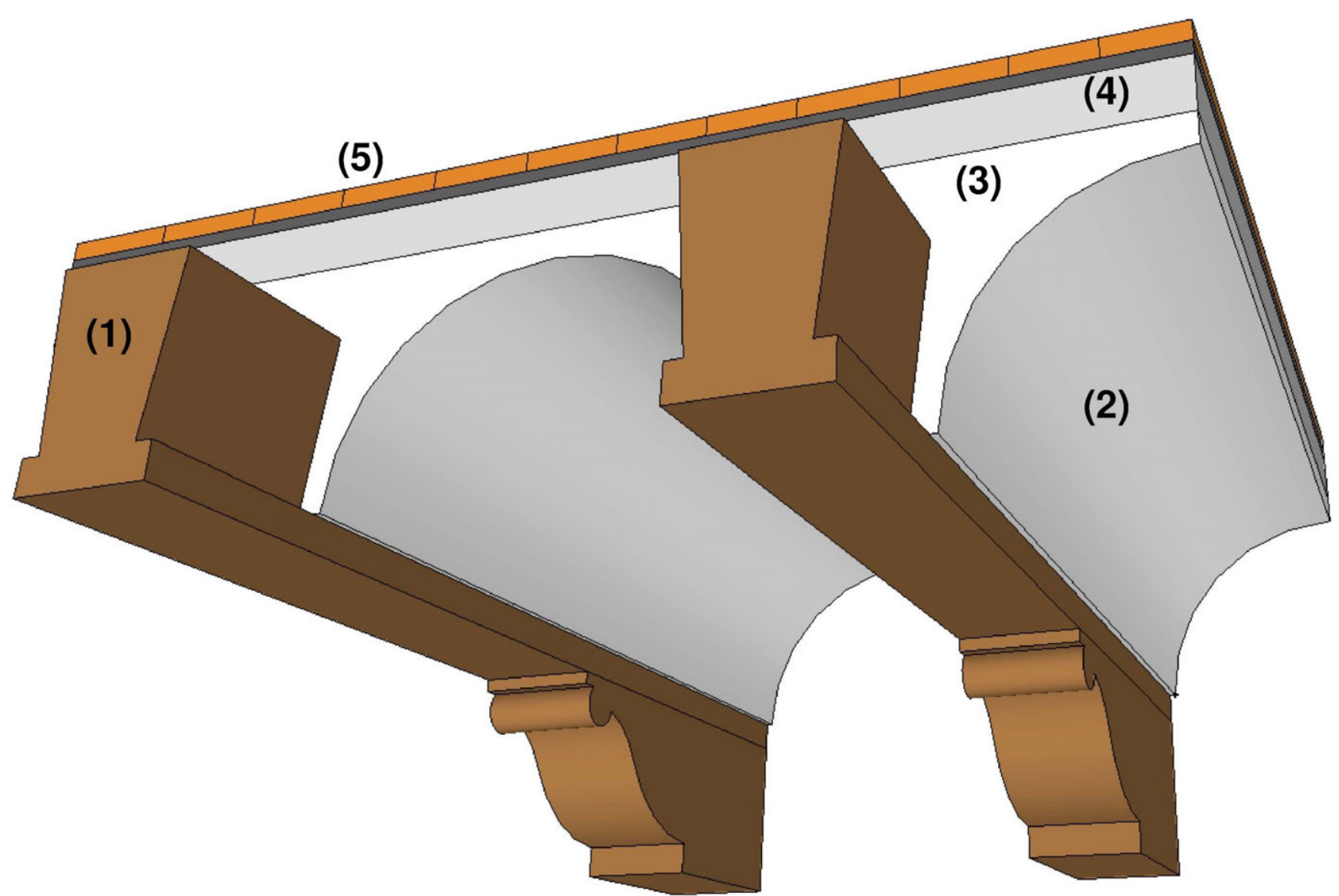

Figura 2. Sección constructiva del forjado en su estado previo a la intervención.

tomada con mortero de cemento, alcanzando un espesor total de $5 \mathrm{~cm}$ (5 en Figura 2).

La solera de hormigón no tenía conexión alguna con la viguería, por lo que constituía únicamente un peso muerto. Podemos deducir que el objetivo de esta intervención fue el de nivelar el suelo de la sala sobre el forjado, pues ya presentaría una flecha incipiente aunque entonces no se estimó que fuera grave. El mallazo confería a la solera un cierto monolitismo al que se confió el reparto de las cargas de las vigas centrales hacia las perimetrales. En el momento previo a la intervención de 2013 el forjado tenía una estructura de sostén (Figura 4), realizada con perfiles metálicos, debidamente ejecutada y arriostrada, y que apoyaba en la solería de planta baja, pues el forjado en su conjunto presentaba una deformación extraordinaria, de entre 8 y $9 \mathrm{~cm}$.

Durante el desmontaje de los rellenos superiores y las operaciones de refuerzo y reparación de las vigas no se observaron lesiones ni ataques biológicos en la madera.

\section{SOLUCIÓN PROPUESTA}

Una escasa escuadría de madera en relación a la luz existente y la carga que había ido adquiriendo a lo largo de los años provocaron una flecha excesiva, a pesar del buen estado de conservación de la madera - salvo en tres vigas fracturadas-. Por ello se planteó reforzar el forjado, manteniendo la madera original procurando respetar su valor material y funcional, y usando en lo posible las técnicas menos invasivas para mejorar el comportamiento del material. La solución propuesta pasaba por aligerar el forjado de sobrecargas en lo posible y aumentar la sección resistente mediante madera de aporte, encolada y fijada a la anterior. Se consideró esta solución la más compatible con los materiales presentes, a la vez que se respondía al requisito de reversibilidad y calidad de la madera (17).

$\mathrm{Al}$ aumentar la sección, el suplemento sólo absorbe una fracción de las nuevas cargas (cargas permanentes de la solería y sobre cargas de uso). Esta fracción es proporcional a la rigidez relativa del suplemento frente al total, y como es sabido, en una sección compuesta, tiene un valor [1]:

$$
Q_{\text {supl }}=Q_{\text {total }} \cdot \frac{\left(E A d^{2}\right)_{\text {supl }}+(E I)_{\text {supl }}}{\left(E A d^{2}\right)_{\text {supl }}+(E I)_{\text {supl }}+\left(E A d^{2}\right)_{\text {vieja }}+(E I)_{\text {vieja }}}
$$

Donde:

- (...) $)_{\text {sup }}$, referido al suplemento.

- (...) vieja $^{\text {, }}$ referido a la madera vieja.
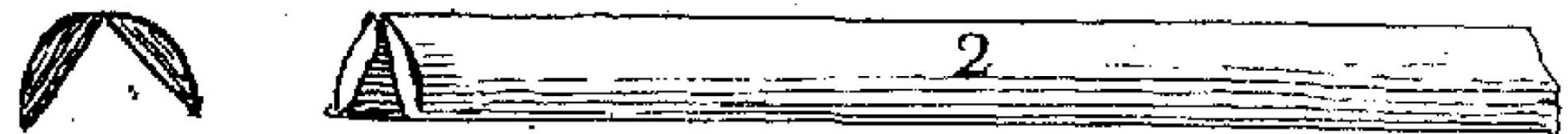

Figura. 3. Formaleta (tomado de Juan de Villanueva, Arte de albañilería, 1827, lám. IX, fig. 2). 


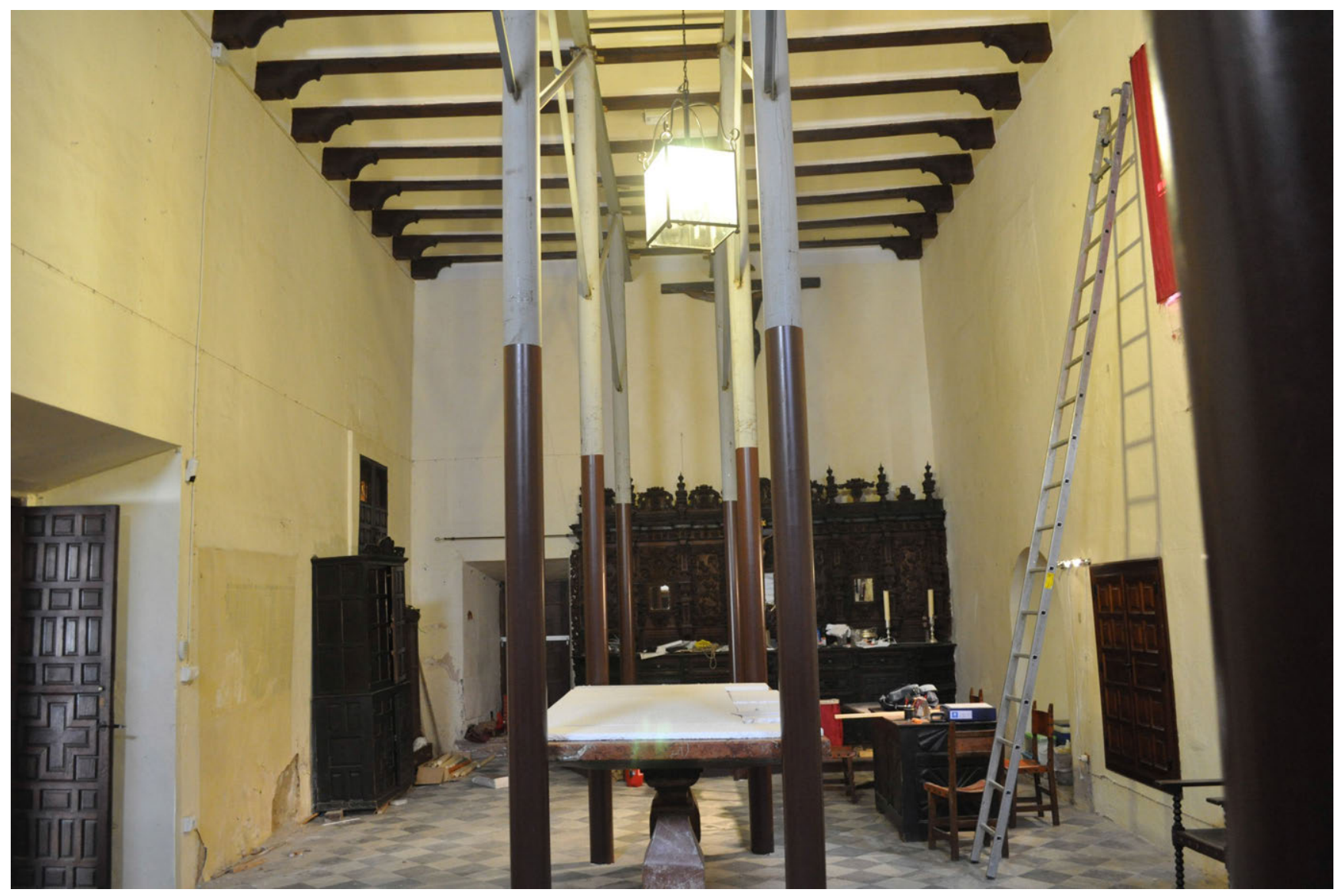

Figura 4. Vista inferior del forjado con el apuntalamiento, justo antes de la intervención.

- $\boldsymbol{Q}_{\text {supl }}$, fracción de la carga que recibe el suplemento.

- $\boldsymbol{Q}_{\text {total }}$, carga total.

- $\boldsymbol{E}$, módulo de elasticidad de cada material, suplemento, o madera vieja.

- $\boldsymbol{A}$, área de cada sección de material.

- I, inercia de cada sección de material.

- $\boldsymbol{d}$, distancia del centroide de cada sección al centroide de la sección compuesta.

El problema principal que aquejaba al forjado era la sección insuficiente de la madera, escasa incluso para soportar su peso propio sin tensiones excesivas ni deformaciones exageradas a largo plazo. Era necesario descargar la madera antigua y conseguir la mayor eficacia del refuerzo. Una alternativa para reducir las tensiones presentes en la madera antigua era aplicar un refuerzo con gran sección. Pero esta opción era poco económica y difícilmente viable, ya que según se muestra en la Figura 2 el espacio por encima de las vigas no era suficiente para un refuerzo adecuado.

La opción elegida finalmente fue la pre-flexión del refuerzo aprovechando el apuntalamiento existente. Con ello se conseguía un uso óptimo de ambos materiales - refuerzo y madera antigua - ya desde un primer momento. Esto permitía reducir la cantidad de refuerzo, al distribuir las cargas de manera más eficaz entre madera vieja y nueva.

Sobre las vigas originales (1 en Figura 5) se disponen los refuerzos encolados ( 6 en Figura 5) unidos además con tirafondos (7 en Figura 5). El relleno que forma las bovedillas (3 en Figura 5) aparece parcialmente desmontado para acceder a la reparación.
Para conseguir un resultado óptimo del refuerzo, el forjado se terminó con soluciones ligeras: sobre el relleno y las vigas reforzadas se dispuso un relleno de arcilla expandida (8 en Figura 5) con densidad menor de $500 \mathrm{~kg} / \mathrm{m}^{3}$, un tablero contrachapado estructural de $22 \mathrm{~mm}$ de espesor (9 en Figura 5), una capa de aislamiento acústico de polietileno celular y finalmente una tarima flotante de madera de $20 \mathrm{~mm}$ de espesor (10 en Figura 5).

\section{DESCRIPCIÓN DETALLADA DEL REFUERZO}

Para el cálculo se adoptaron los siguientes Documentos Básicos del Código Técnico de la Edificación (18): DB-SE-A, DB-SE-AE, DB-SE-M y DB-SE-SI, que actualmente rigen el ámbito constructivo español.

Para las vigas de madera antiguas caracterizadas como pino silvestris se adoptó una resistencia característica a la flexión de $18 \mathrm{~N} / \mathrm{mm}^{2}$ y para el resto de propiedades mecánicas, las propias de una clase estructural $\mathrm{C}-18$. Aunque las vigas, de grandísima calidad, habían demostrado tener una resistencia muy superior a la estimada.

Sobre las vigas existentes se añadió un refuerzo de madera laminada encolada de clase resistente GL-24 h, de $17 \times 17 \mathrm{~cm}^{2}$ (6 en la Figura 5).

Dadas las condiciones interiores se aplicó una clase de servicio 1 , y una clase de duración de la carga media correspondiente a las sobrecargas de uso.

Ambas maderas, vigas y refuerzo, se unieron con resina epoxi bicomponente apta para uso estructural (19), adecuada para 


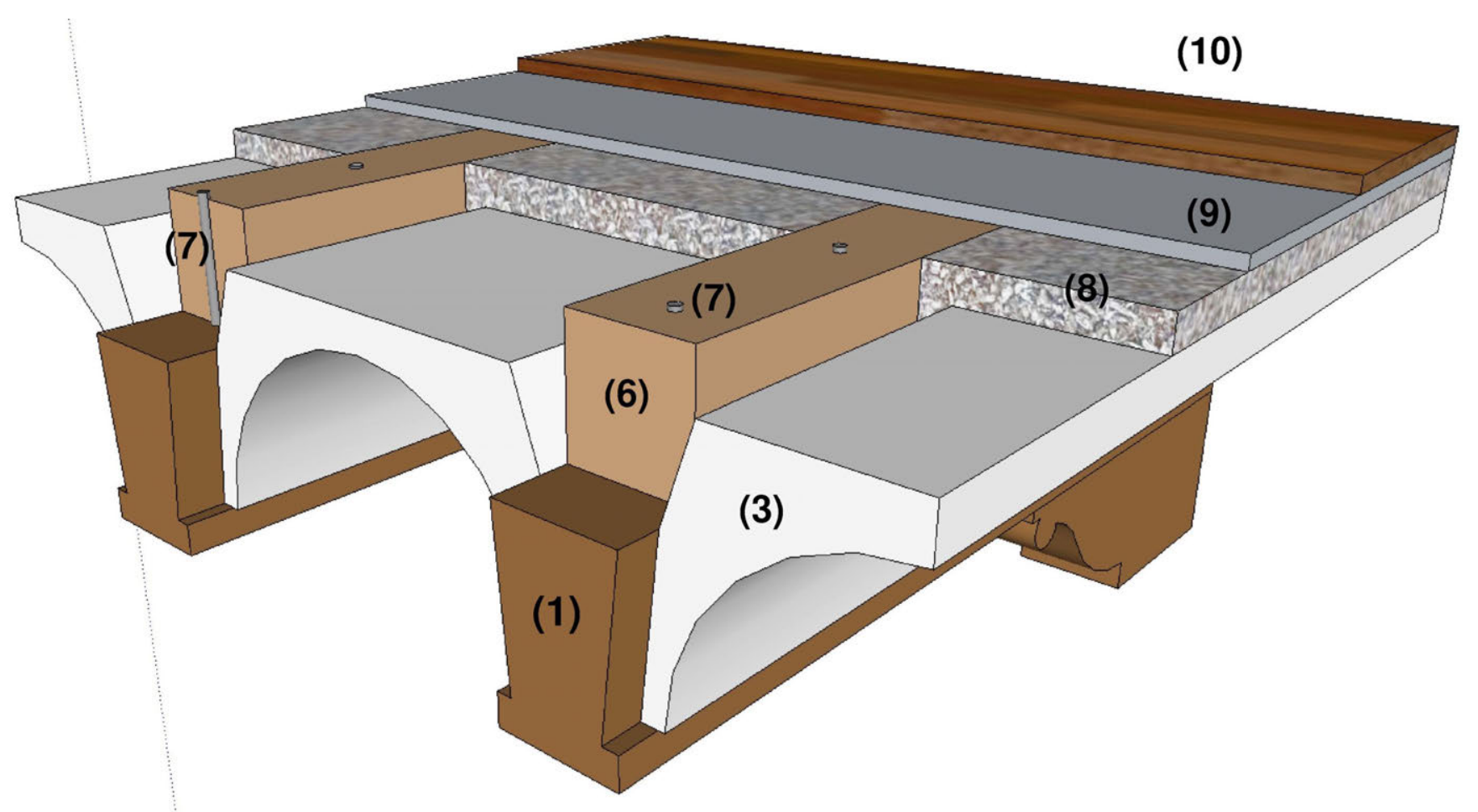

Figura 5. Sección constructiva del estado final, ya reforzado.

una capa gruesa y con resistencia a la tracción declarada de $18-24 \mathrm{~N} / \mathrm{mm}^{2}$ y cortante de $>6 \mathrm{~N} / \mathrm{mm}^{2}$ (20). Entre ambos elementos se emplearon tirafondos de conexión de $10 \times 250$ mm según DIN 571, dispuestos cada 300 mm.

Al uso de la sala, expositivo, le correspondía inicialmente la subcategoría de uso C3 (DB-SE-AE) (15). Sin embargo, de acuerdo con los supuestos de incompatibilidad del mismo Código, puesto que se trata de una rehabilitación, se podrían aplicar «aquellas soluciones que permitan el mayor grado posible de adecuación efectiva». Como resultado se obtuvo una limitación de uso de $3 \mathrm{kN} / \mathrm{m}^{2}$ y una limitación de aforo de 100 personas, perfectamente compatible con el uso previsto.

\subsection{Explicación sucinta del proceso}

Debido a la pre-flexión, la explicación del refuerzo no se puede separar de la del proceso de ejecución. La Figura 6 resume algunos de los estados del proceso, una vez realizadas las labores iniciales de desmontado de la solería y de los rellenos y efectuada la limpieza. En A) se representa la colocación del refuerzo superior. B) representa la unión provisional y el pretensado de las piezas mediante gatos más el apuntalamiento inferior de la madera antigua, en este momento se encolan las piezas. En C) se representa el estado final en el cual se eliminan los apoyos y los gatos; entonces el refuerzo mantiene levantadas las vigas antiguas, a la vez que alcanza el nivel de trabajo establecido.

Siguiendo la misma figura, $\boldsymbol{d}_{\mathbf{1}}$ representa la flecha inicial de la madera antigua $-\mathrm{A}$ ) una vez eliminadas una parte de las cargas permanentes-. En la fase B) la flecha $\boldsymbol{d}_{2}$ es menor por la deformación inversa realizada con el apeo y los gatos; esta deformación permite acercar la madera de refuerzo a la madera antigua. En la fase C), y actuando las cargas finales, la flecha $\boldsymbol{d}_{3}$ es algo mayor que $\boldsymbol{d}_{2}$ pero menor que $\boldsymbol{d}_{\boldsymbol{1}}$.

\subsection{Cálculo}

En el cálculo, de acuerdo el DB-SE-M y el Eurocódigo 5 (21), se estudiaron las diferentes fases para evitar que en ninguna de ellas se sobrepasaran las tensiones límites admisibles para ambos materiales. En las fases en las que se había consolidado la unión la comprobación se hizo bajo la hipótesis de unión perfecta entre ambos materiales, en lógica por la unión encolada, con la sección homogeneizada a las propiedades de la madera antigua. Se aplicó el principio de superposición de estados de una fase a otra, sumando las tensiones de cada fase a la siguiente.

Fue necesario desarrollar una herramienta de cálculo específica que incorporara esta actuación y que facilitara la toma de decisiones viga a viga. Se introducían los datos correspondientes a cada fase: sección de la madera antigua y sus propiedades, sección del suplemento y sus propiedades, tipo de carga y su situación a lo largo de la viga -concentrada o repartida-, deformación inicial y la deformación inducida. En el estado inicial trabaja con dos secciones independientes, y a partir de la fase de unión trabaja con una sección única.

Los gráficos de la Figura 7 muestran en abscisas las tensiones de trabajo de las secciones antes y después de la intervención. En ordenadas se muestra la distancia de la fibra a la cara inferior. El tramo superior se corresponde con el refuerzo de madera laminada encolada, y el inferior con las vigas antiguas. La Tabla 1 muestra los valores numéricos.

La «Situación inicial» mostrada en la Figura 7 se corresponde con la situación A) de la Figura 6 donde la madera de aporte no se ha colocado o no se ha conectado todavía. En la gráfica la línea de trazos muestra las tensiones iniciales de la madera antigua antes de eliminar la solería y los rellenos, y la línea continua las tensiones una vez eliminadas estas cargas. Estas tensiones se corresponden en la Tabla 1 con las columnas «cargas iniciales»y «losa desmontada». 
A)

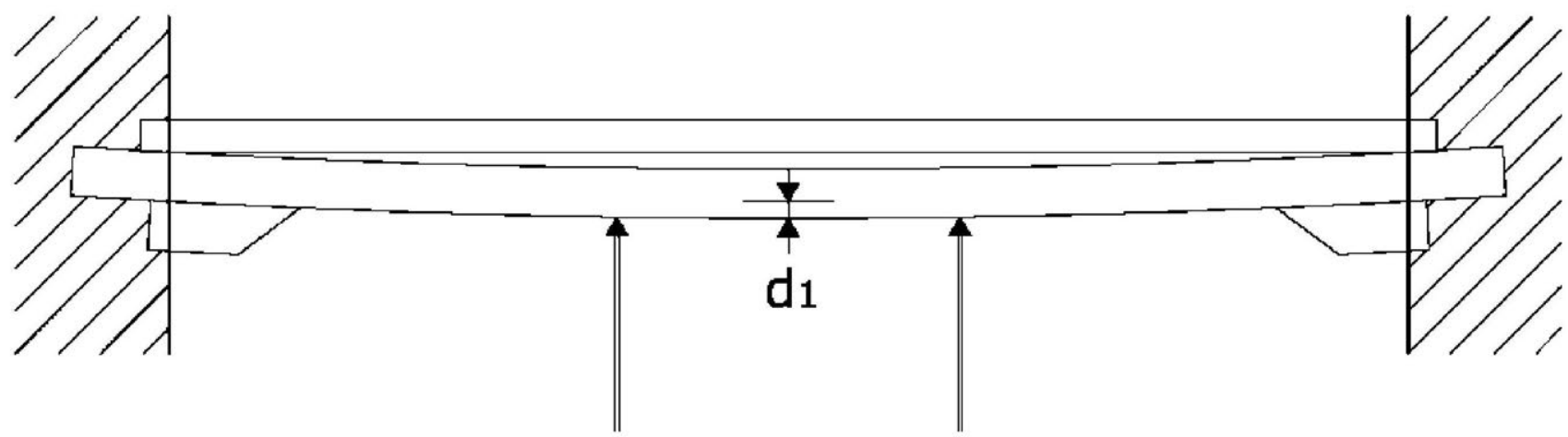

B)

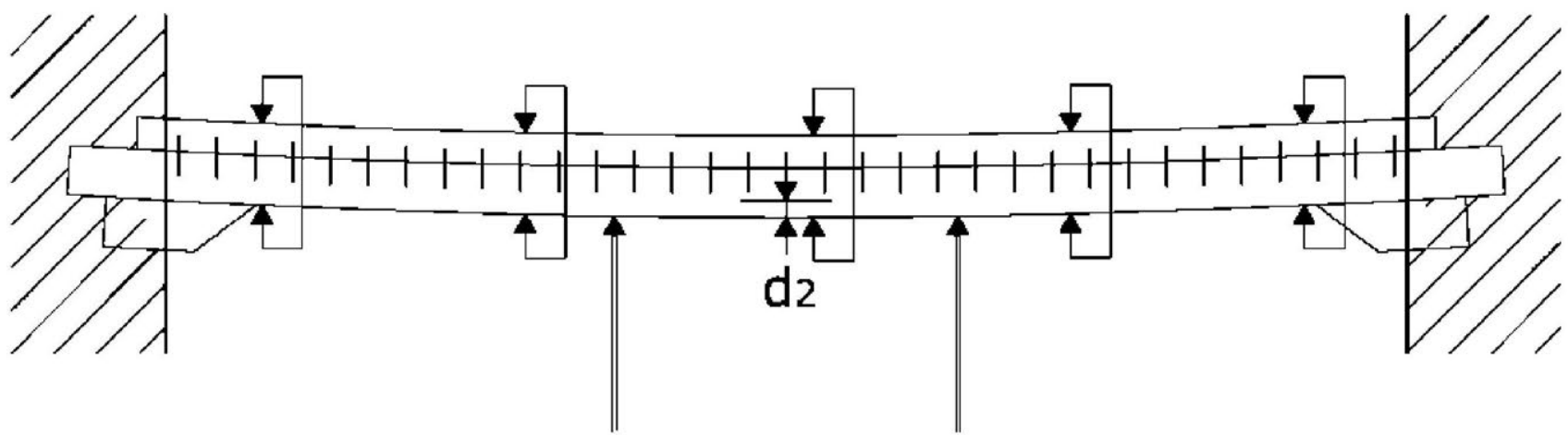

C)

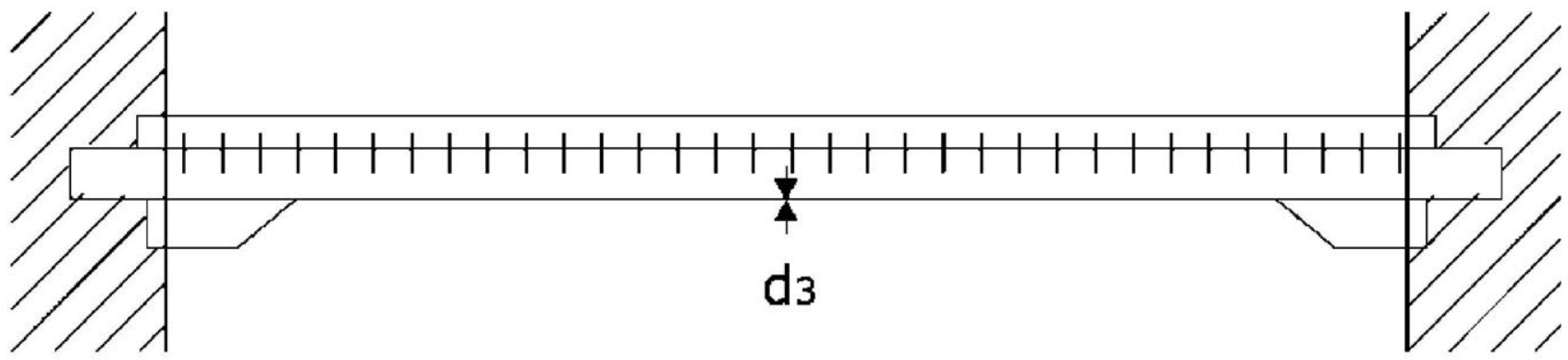

Figura. 6. Proceso resumido de pre-flexión.

Siguiendo la misma gráfica y tabla, «pre-flexión» muestra las tensiones de ambos materiales, de signo opuesto, debido al apriete mutuo y al ejercido por el apeo inferior sobre las vigas originales. Se corresponde con el caso B) de la Figura 6.

El estado «unión» muestra las tensiones de la sección compuesta, con la unión encolada endurecida, y una vez retirados los gatos y el apoyo inferior.

El estado final «unión, cargas finales» representa las tensiones una vez terminado de construir el relleno y solería, y con las cargas de uso previstas. En la Tabla 1 se ha añadido una columna más a la derecha, expresando estas tensiones en for- ma de índices de trabajo - valores de cálculo mayorados-. Se observa que la madera antigua ha quedado muy descargada en contra de la madera de aporte - caso C) de la Figura 6.

Cuando se produce la unión efectiva de ambos materiales, al soltar los aprietes, aparecen tensiones de despegue entre ambas piezas. Aun cuando el encolado tenía capacidad sobrada para soportar estas tensiones, se procedió a colocar una serie de tirafondos en perpendicular, y penetrando en ambos materiales, como medida de seguridad extra.

Recordamos que con la pre-flexión se pretendían dos objetivos principales: reducir la flecha y conseguir el máximo tra- 


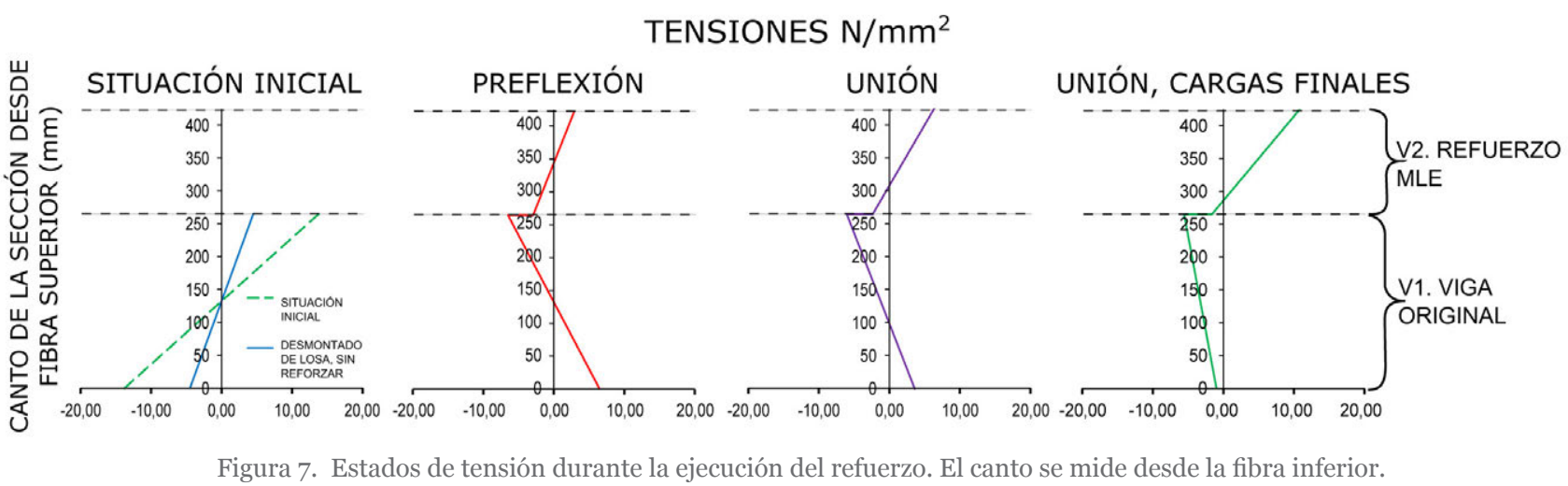

Tabla 1. Tensiones $\left(\mathrm{N} / \mathrm{mm}^{2}\right)$ e índices de trabajo durante las fases de ejecución (sin mayorar excepto los índices de trabajo, columna señalada con "*")

\begin{tabular}{|c|c|c|c|c|c|c|c|}
\hline \multicolumn{2}{|c|}{$\begin{array}{c}\text { Profundidad de la } \\
\text { fibra (mm) }\end{array}$} & $\begin{array}{c}\text { Situación } \\
\text { inicial cargas } \\
\text { iniciales }\end{array}$ & $\begin{array}{c}\text { Losa } \\
\text { desmontada }\end{array}$ & Pre-flexión & Unión & $\begin{array}{c}\text { Unión, cargas } \\
\text { finales }\end{array}$ & $\begin{array}{c}\text { Unión, cargas } \\
\text { finales. Indices } \\
\text { de trabajo (*) }\end{array}$ \\
\hline \multirow{2}{*}{ Suplemento } & 425 & - & - & 2,970 & 6,366 & 11,276 & $96 \%$ \\
\cline { 2 - 8 } & 265 & - & - & $-2,970$ & $-2,298$ & $-1,326$ & $-11 \%$ \\
\hline \multirow{2}{*}{ Viga antigua } & 265 & 13,80 & 4,53 & $-6,60$ & $-6,08$ & $-5,32$ & $-63 \%$ \\
\cline { 2 - 8 } & 0 & $-13,80$ & $-4,53$ & 6,60 & 3,62 & $-0,69$ & $-8 \%$ \\
\hline
\end{tabular}

bajo de ambos elementos unidos, descargando a su vez la madera antigua de parte de su carga. En la Figura 8 se comparan los índices de trabajo que se han obtenido con la pre-flexión y los que se hubieran obtenido sin esta deformación previa. Los mismos valores se recogen en la Tabla 2. Si no se hubiera aplicado la pre-flexión, los índices de trabajo a flexión de la madera antigua (referidos a la clase resistente asignada) hubieran superado el $100 \%$ (línea discontinua) y el refuerzo hubiera quedado infrautilizado con índices de trabajo muy pobres (42\%). La deformación previa corrige e invierte estos términos y aprovecha al máximo el refuerzo.

\subsection{Comportamiento ante el incendio}

Es conocida la debilidad de los encolados de resina epoxi con las altas temperaturas presentes en un fuego. Sin embargo, es una cuestión que, al menos hasta hace relativamente poco, se dejaba de lado en las restauraciones monumentales por la complicación extra que suponía en un trabajo que ya tiene bastante de experimental y de arriesgado en demasiados casos.

Por el contrario, esta estructura posee una seguridad doble en el caso de incendio. Por un lado, la línea de encolado está protegida por una bovedilla de yeso con espesor de al menos $5 \mathrm{~cm}$, más un relleno de arcilla expandida. Estas condiciones son suficientes para salvaguardar el encolado el tiempo necesario. Por otro lado, aun en el caso de que fallara el encolado -situación muy improbable-, la magnitud de las cargas no llegaría a dañar ambas maderas, antigua y de aporte, aun teniendo en cuenta la pérdida de sección aplicable a la madera antigua calculada de acuerdo con el método de la sección reducida (DB-SI anejo E) (18).

\section{DESCRIPCIÓN DETALLADA DEL PROCESO DE EJECUCIÓN}

Antes de la intervención el forjado presentaba una flecha superior a $5 \mathrm{~cm}$ en algunas vigas, a la par que estaba sustentado por un apuntalamiento (Figura 4). En este momento gravita-
Tabla 2. Comparativa entre los índices de trabajo finales con y sin pre-flexión.

\begin{tabular}{|c|c|c|c|}
\hline \multicolumn{2}{|c|}{$\begin{array}{c}\text { Profundidad de la fibra } \\
\text { (mm) }\end{array}$} & $\begin{array}{c}\text { Con } \\
\text { pre-flexión }\end{array}$ & $\begin{array}{c}\text { Sin } \\
\text { pre-flexión }\end{array}$ \\
\hline \multirow{2}{*}{ Suplemento } & 425 & $96 \%$ & $42 \%$ \\
\cline { 2 - 4 } & 265 & $-11 \%$ & $8 \%$ \\
\hline \multirow{2}{*}{ Viga antigua } & 265 & $-63 \%$ & $62 \%$ \\
\cline { 2 - 4 } & 0 & $-8 \%$ & $-104 \%$ \\
\hline
\end{tabular}

ba sobre el mismo la carga inútil de una solera de hormigón (4 en Figura 2).

El refuerzo del forjado formaba parte de una intervención sobre esta zona de la iglesia, que estaba aquejada, entre otros problemas, de humedades por falta de drenaje en el subsuelo. Uno de los síntomas más llamativos era el enorme abultamiento que tenían las solerías de la planta baja, perfectamente observable a simple vista. Por ello una de las actuaciones sobre la iglesia fue proceder al drenaje del subsuelo. Estos trabajos se realizaron a la vez que se procedía al refuerzo del forjado, lo que provocó un descenso notable de los abultamientos, que incidió a su vez en el descenso del apuntalamiento que sostenía el forjado. En esos momentos el forjado quedó separado del apeo escasos centímetros, que aprovechamos para medir la flecha real de cada una de las vigas, llegándose a medir hasta $9 \mathrm{~cm}$ de deformación máxima en algunas vigas, y que se corresponde con la máxima deformación estimada a largo plazo.

Se levantó la solería de la planta primera (5 en Figura 2) y se demolió la losa de hormigón (4 en Figura 2) que gravitaba sobre el forjado, previamente cortada en bloques, y que dejó a la vista el relleno original que tapaba las maderas (Figura 9). Tras esta operación se midió de nuevo la flecha de las vigas.

Una vez realizados estos desmontajes, únicamente se pudo retirar una parte del relleno que cubría las vigas y despejar los empotramientos en los muros (sólo entonces se pudo realizar una inspección en profundidad de las partes ocultas de la ma- 


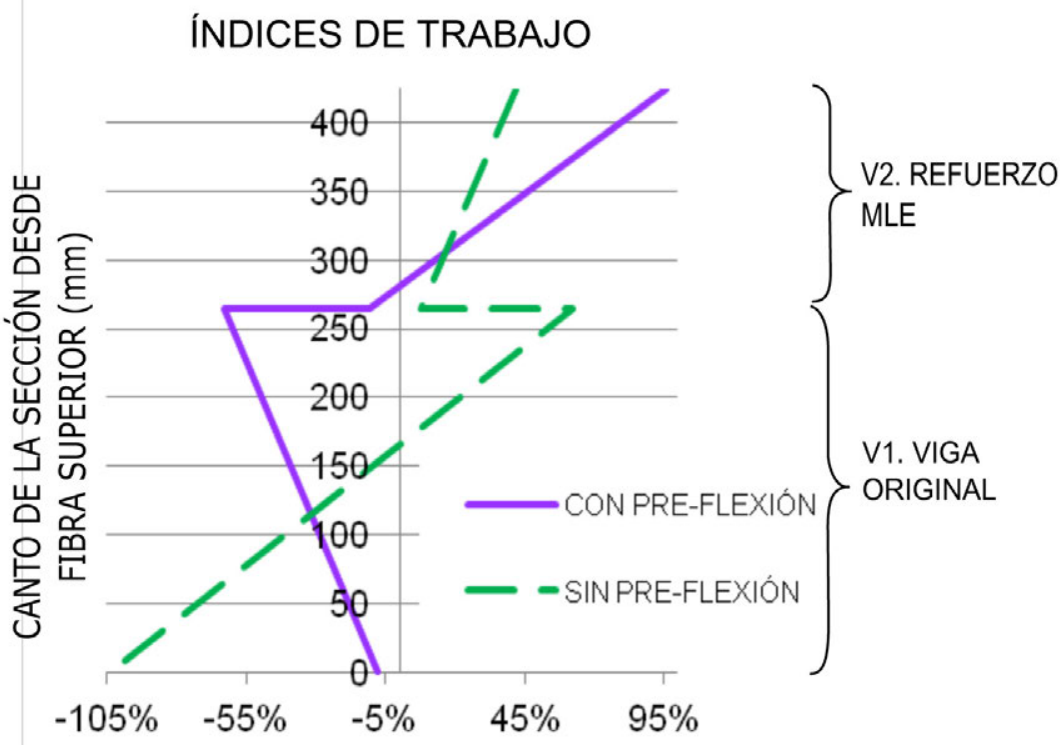

Figura 8. Comparación entre el refuerzo con y sin pre-flexión. Índices de trabajo.

dera), comprobando el excelente estado de conservación de la madera según se observa en la Figura 9, tanto en los empotramientos como en la cara superior y laterales, distinguiendo incluso las marcas de montaje talladas por los carpinteros. En este momento se comprobó la naturaleza del relleno (3 en Figura 2), que forma un continuo con las bovedillas, y se diferencia sólo en que es un material con más cascajo y menos conglomerante (de acuerdo a lo establecido por Juan de Villanueva). Como resultado de ello sólo fue posible eliminar el relleno situado justo por encima de las vigas, para no destruir las bovedillas. Además, la densidad de este relleno resultó ser de $1.710 \mathrm{~kg} / \mathrm{m}^{3}$, cifra intermedia entre los dispares valores de 1.200 a $2.800 \mathrm{~kg} / \mathrm{m}^{3}$ que establece el BD-SE-AE (18). Todo ello tuvo consecuencias en el cálculo.

Las vigas antiguas fueron posteriormente calzadas mediante acuñados sobre el apeo inferior, lo que permitió en muchas de ellas recuperar ya parte de la flecha, al estar ahora descargado de los sobrepesos.

En esta fase se procedió también a perforar las bovedillas en 5 puntos (tres intermedios y dos extremos) a lo largo de las vigas para que pasaran los gatos de apriete de la fase siguiente. Estas perforaciones ayudaron también a recuperar parte de la deformación, ya que las bovedillas ejercen un efecto arco que también es considerable en el sentido longitudinal, ya que coarta la flexión de la madera unida a ellas. Las tensiones provocadas por esta fase de apriete eran de signo contrario a las habituales en las vigas de madera, y contrarrestaban las tensiones debidas al peso, nada despreciable aún, de las bovedillas.

Como vemos, fue fundamental para el éxito de esta operación la presencia no buscada de un apuntalamiento inferior en excelentes condiciones y con gran capacidad, y que además disponía dos carreras perpendiculares a las vigas, por tanto, colocadas en una situación inmejorable para nuestros propósitos.

Una vez realizados estos trabajos, y limpio el trasdós, se preparó la superficie superior de las mismas que habían quedado a la vista y que iban a recibir el encolado, mediante una previa aspiración y limpieza exhaustiva (Figura 9).

Una vez preparada la madera antigua, se colocaron los refuerzos de madera laminada, se ubicaron los gatos y se apretaron

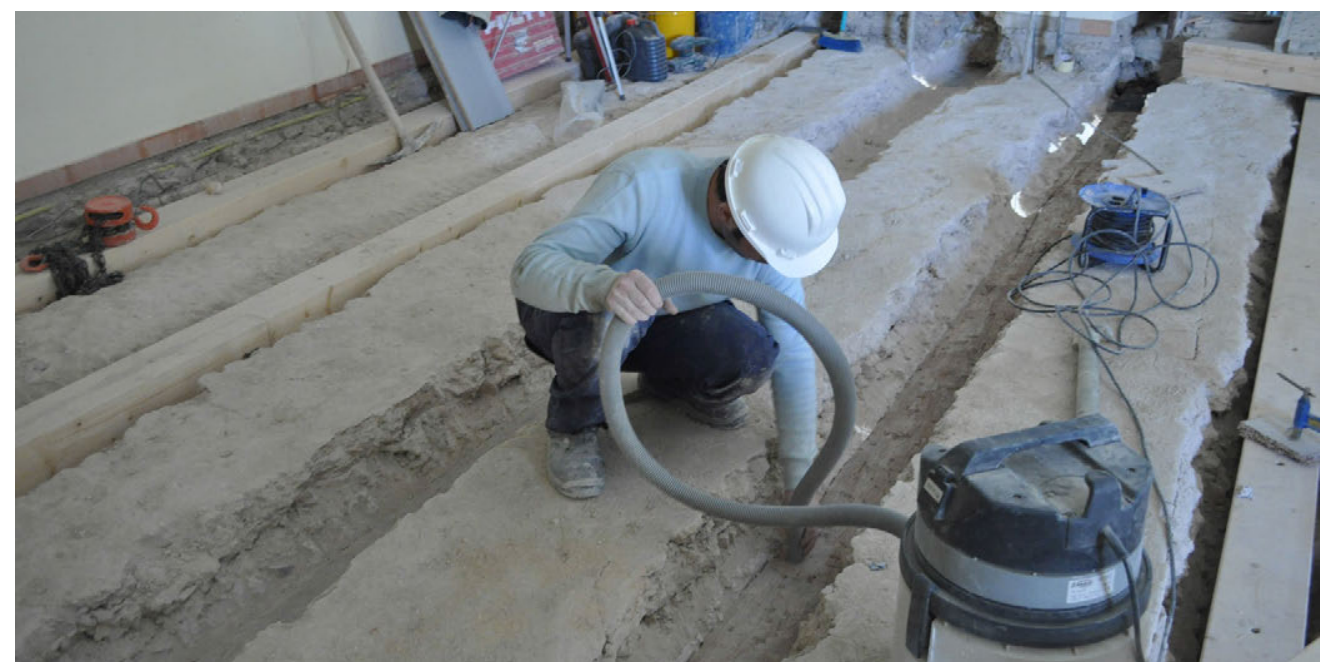

Figura 9. Trabajo de limpieza de las vigas de madera antes de su encolado. Se puede observar el cajeado abierto en las bovedillas para la colocación de los refuerzos, y las vigas ya reforzadas. 


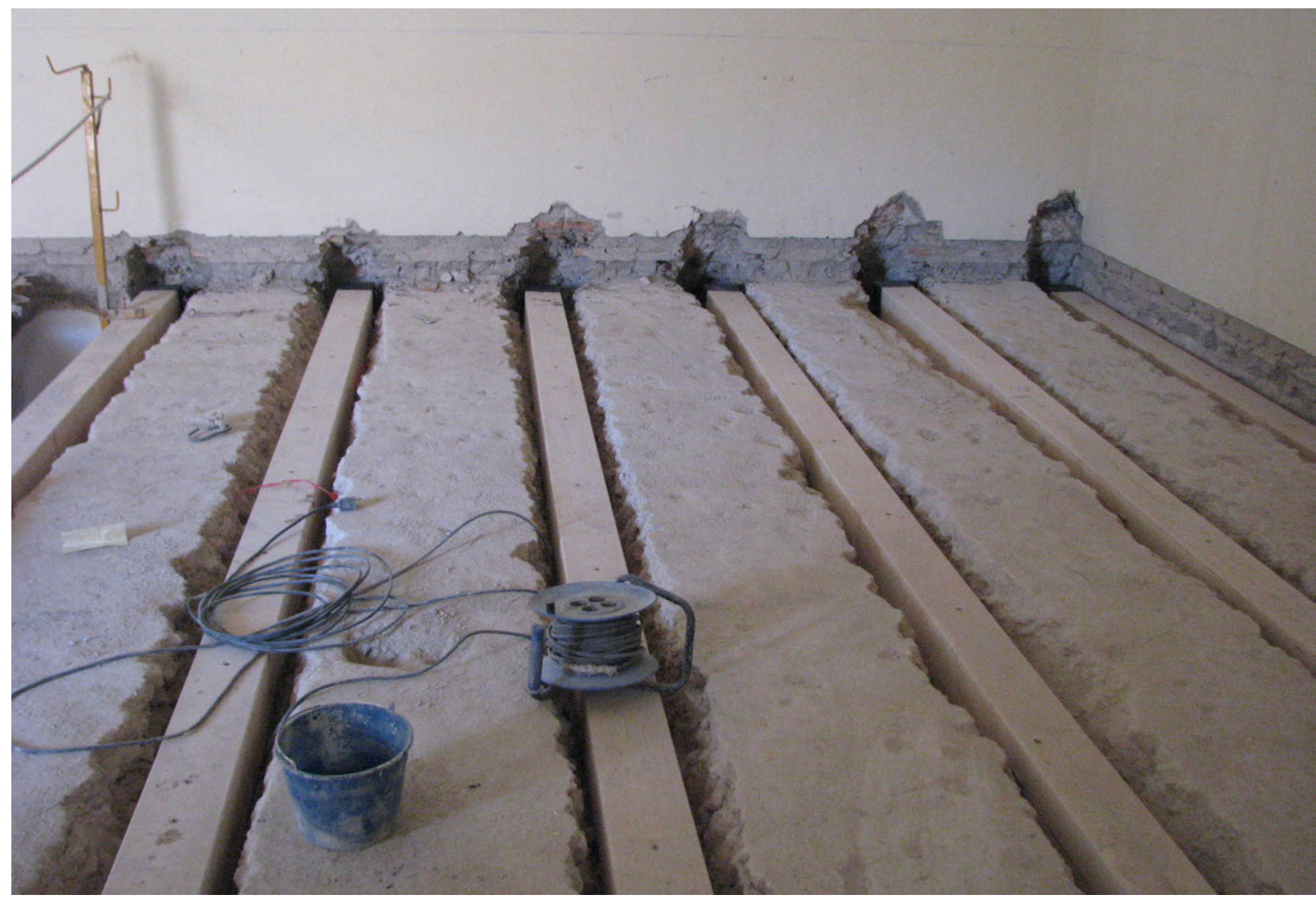

Figura 10. Forjado reforzado con los refuerzos ya encolados.

ambas piezas (madera antigua y laminada) sin llegar al límite máximo de deformación establecido ( $45 \mathrm{~mm}$ ). Este límite evitaba tensiones excesivas en ambos materiales. Antes de llegar al contacto, se procedió a encolar con resina epoxi de formulación adecuada para rellenar las irregularidades. Inmediatamente después se apretó por completo hasta conseguir el contacto entre refuerzo y madera vieja. Esta operación se completó disponiendo una serie de conectores como seguridad extra para soportar las tracciones en la junta encolada.

Una vez endurecido el encolado, se procedió a soltar los gatos y las cuñas inferiores. En este momento las vigas quedaron prácticamente rectas, con una deformación menor de $2 \mathrm{~cm}$ (Figura 10). Finalmente, una vez concluido el refuerzo se procedió a realizar el relleno de arcilla expandida hasta conseguir el nivel, para posteriormente colocar un tablero y sobre él un suelo de tarima flotante de madera.

\section{CONCLUSIONES}

Aun cuando hay técnicas desarrolladas para la rehabilitación de forjados de madera, en cada intervención existen unos condicionantes constructivos tanto del propio forjado como del resto del edificio, de ejecución, económicos y de diverso tipo, que hacen singular cada obra. La intervención que hemos expuesto hubiera sido muy diferente, dependiendo de la configuración del forjado en su estado inicial, y de aspectos más variopintos, como los niveles de suelo actuales, el tipo de relleno, la existencia de un apuntalamiento previo o la aplicación errónea de un falso refuerzo previamente a nuestra intervención.
En esta experiencia se demuestra la capacidad de aprovechamiento de la madera antigua como elemento estructural, que va mucho más allá de los límites y etiquetas que habitualmente le solemos atribuir. La reacción habitual de un técnico - preocupado por lógica por la seguridad de la construcciónante un forjado con unas deformaciones de la magnitud de las observadas hubiera sido el rechazo y sustitución de la madera por otro material o por otro sistema nuevo de forjado. Sin embargo, pese a que las tensiones presentes en las vigas antiguas superaban con mucho nuestras estimaciones respecto de la seguridad, esta madera estaba, en general, en unas condiciones inmejorables. Hemos demostrado además la capacidad que posee la madera de recuperar gran parte de la deformación.

A lo largo del trabajo ha quedado patente cómo las incorrectas intervenciones sobre el patrimonio -en este caso mediante la colocación sobre el forjado de una losa de hormigón, sin conexión y mal armada- provocan problemas aún mayores de los que pretenden resolver. En este sentido son innumerables los ejemplos que se podrían citar. Este trabajo es, por tanto, un ejemplo que muestra la posibilidad de eliminación y corrección de estas intervenciones.

Por último queremos señalar que la aplicación de suplementos de madera, unidos mediante encolado a las piezas originales, combinadas con la pre-flexión, es un sistema que permite un refuerzo lógico, con mayor eficacia, menor impacto, y que permite en gran medida perpetuar la lógica del sistema estructural original de acuerdo con las consideraciones más aceptadas. 


\section{REFERENCIAS}

(1) Gómez Sánchez, I. (2006). Las estructuras de madera en los tratados de arquitectura (150o-1810), p. 6. Madrid: AITIM, Asociación de Investigación Técnica de las Industrias de la Madera.

(2) Alonso, M. J. (2001, 17-21 julio). La conservación de los bienes culturales en madera. Nuevas orientaciones. En Iglesias Gil, J. M. (Ed.), Cursos sobre el patrimonio histórico: Actas de los XI Cursos Monográficos sobre el Patrimonio Histórico (pp. 103-113). Reinosa: Universidad de Cantabria, Ayuntamiento de Reinosa.

(3) Nuere Matauco, E. (2007). Madera en restauración y rehabilitación. Nota técnica. Informes de la Construcción, 59(506): 123-130, doi: http://dx.doi.org/10.3989/ic.2007.v59.i506.

(4) Bestraten, S., Hormias, E. (2012). Consolidación estructural del edificio patrimonial de C/Ripoll, 25 de Barcelona - Ca la Dona. Informes de la Construcción, 64(n. ${ }^{0}$ extra): 79-94, doi: http://dx.doi.org/10.3989/ic.11.051.

(5) ICOMOS (2003). Principios para el análisis, conservación y restauración de las estructuras del patrimonio arquitectónico. Ratificada por la $14 \cdot{ }^{\mathrm{a}}$ Asamblea General del ICOMOS, Octubre. Victoria Falls, Zimbabwe. http://www.icomos.org/ charters/structures_sp.pdf

(6) Landa Esparza, M. (1999). Nuevas técnicas de reparación de estructuras de madera. Elementos flexionados. Aporte de madera-Unión encolada II. Metodología de puesta en obra. RE: revista de edificación (29): 30-36.

(7) Arriaga, F., Peraza, F., Esteban, M., Bobadilla, I., García, F. (2002). Intervención en estructuras de madera, p. 151. Madrid: AITIM, Asociación de Investigación Técnica de las Industrias de la Madera.

(8) Wangaard, F. F. (1964). Elastic deflection of wood-fiberglass composite beams. Forest Products Journal, 14(6): 256260.

(9) Borri, A., Corradi, M., Grazini, A. (2004). A method for flexural reinforcement of old Wood beams with CFRP materials. Composites Part B: Engineering, 36(2): 143-153, doi: http://dx.doi.org/10.1016/j.compositesb.2004.04.013.

(10) Triantafillou, T. C., Deskovic, N. (1992). Prestressed FRP sheets as external reinforcement of wood members. Journal of Structural Engineering, 118(5): 1270-1284.

(11) Lipski, R. Procedimiento de fabricación de vigas con armadura rígida pretensada. España: ESo370273 A1, (B28B7/o8; B28B7/38), Po370273, 6 agosto 1969, 18 pp.

(12) Morón de Castro, M. F. (1995). La iglesia de San Miguel de Morón. Cinco siglos en la historia de Morón de la Frontera. $X I V-X V I I I$, pp. 119, 133 y 150, Sevilla: Universidad de Sevilla [etc.].

(13) Fernández Naranjo, J. A. (2004). La iglesia de San Miguel arcángel de Morón de la Frontera. Síntesis de arquitecturas (tesis doctoral no publicada). Sevilla: Universidad de Sevilla.

(14) Morilla Cala, J. P. (2006). En el V Centenario de la reconstrucción de la iglesia de San Miguel. El plano, el texto y la añeja fotografía. Sevilla.

(15) Guerrero Vega, J. M., Pinto Puerto, F. (2013). Informe final de obras de intervención en sacristía y dependencias anexas. Anexo 1. (informe profesional inédito). Sevilla: Colegio Oficial de Arquitectos de Sevilla.

(16) de Villanueva, J. (1827). Arte de albañilería ó Instrucciones para los jóvenes que se dediquen a él... con diez estampas para su mayor inteligencia, p. 59 y lám. IX. Madrid: Oficina de Don Francisco Martínez Dávila. Facsímil en: http:// bibliotecadigitalhispanica.bne.es, Biblioteca Nacional.

(17) ICOMOS (1999). Principios que deben regir la conservación de las estructuras históricas en madera, ptos. 6 y 9, y 5.b, Ratificada por la 12 Asamblea General del ICOMOS celebrada en México del 17 al 24 de octubre, http://www.icomos. org/charters/wood_sp.pdf.

(18) RD 314/2006, de 17 de marzo, por el que se aprueba el Código Técnico de la Edificación (CTE). Boletín Oficial del Estado, $\mathrm{n} .{ }^{\circ}$ 74, 28 de marzo de 2006 (actualizado 12 de septiembre 2013). Madrid.

(19) Ministerio de Vivienda (2009, abril). Documento Básico SE-M. Seguridad estructural Madera, tabla 4.1. Madrid: Boletín Oficial del Estado.

(20) AENOR-CEN (2012). UNE-EN ISO 527-1:2012. Plásticos. Determinación de las propiedades en tracción. Parte 1: Principios generales. Madrid: Asociación Española de Normalización.

(21) CEN (2006). Eurocode 5. EN 1995-1-1:2006. Design of timber structures. Part 1-1: General - Common rules and rules for buildings. Brussels: Comité Européen de Normalisation (CEN). 